\title{
The First Birkhoff Coefficient and the Stability of 2-Periodic Orbits on Billiards
}

\author{
Sylvie OLIFFSON KAMPHORST and Sônia PINTO DE CARVALHO \\ Departamento de Matemática ICEx UFMG \\ Caixa Postal 702, 30123-970 Brazil \\ syok@mat.ufmg.br, sonia@mat.ufmg.br
}

\begin{abstract}
In this work we address the question of proving the stability of elliptic 2-periodic orbits for strictly convex billiards. Eventhough it is part of a widely accepted belief that ellipticity implies stability, classical theorems show that the certainty of stability relies upon more fine conditions. We present a review of the main results and general theorems and describe the procedure to fullfill the supplementary conditions for strictly convex billiards.
\end{abstract}

\section{Introduction}

Let $\alpha$ be a plane, closed, regular and strictly convex curve. The billiard problem on $\alpha$ consists in the free motion of a point particle in the plane region enclosed by $\alpha$, with unitary velocity and being reflected elastically at the impacts with the boundary. The trajectories are polygonals in the region.

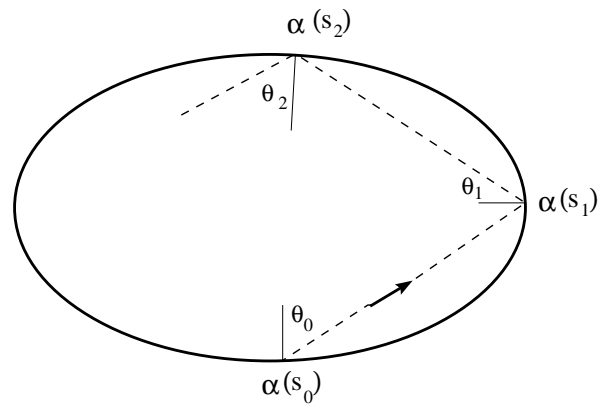

The motion is completely determined by the point of reflection at $\alpha$ and the direction of motion immediately after each reflection. For instance, the arclength parameter $s$, which locates the point of reflection, and the tangential component of the momentum $p=\sin \theta$, where $\theta$ is the angle between the direction of motion and the normal to the boundary at the reflection point, describe the system. Good introductions to billiards can be found in [3], 4], 6], [8], 10, [15] or 16.

The billiard model defines a map $T$ which to each $(s, p)$ in the annulus $\mathcal{A}=[0, L) \times(-1,1)$, representing the pair impact coordinate and direction of motion, associates the next impact and direction:

$$
\begin{aligned}
& T: \mathcal{A} \rightarrow \mathcal{A} \\
& (s, p) \longmapsto(S(s, p), P(s, p))
\end{aligned}
$$


Since the particle can travel along the same polygonal in both senses, the problem is time-reversing and the inverse map $T^{-1}$ is well defined.

The derivative of $T$ at $(s, p)$ is implicitly calculated and is given by the formulae:

$$
\begin{aligned}
\frac{\partial S}{\partial s} & =\frac{l(s, p)-R(s) \cos \theta(p)}{R(s) \cos \theta(P)} \\
\frac{\partial S}{\partial p} & =\frac{l(s, p)}{\cos \theta(p) \cos \theta(P)} \\
\frac{\partial P}{\partial s} & =\frac{l(s, p)-R(s) \cos \theta(p)-R(S) \cos \theta(P)}{R(s) R(S)} \\
\frac{\partial P}{\partial p} & =\frac{l(s, p)-R(s) \cos \theta(P)}{R(S) \cos \theta(p)}
\end{aligned}
$$

where $S$ stands for $S(s, p)$ and $P$ for $P(s, p), l(s, p)$ is the distance between the two consecutive impacts points $\alpha(s)$ and $\alpha(S), R$ is the radius of curvature of $\alpha$ and $\cos \theta(p)=\sqrt{1-p^{2}}$ is the normal component of the momentum.

If $\alpha$ is a $C^{k}$ curve, $k \geq 2$, the billiard model gives rise to a discrete two-dimensional $C^{k-1}$ area preserving dynamical system, whose orbits are given by

$$
\mathcal{O}(s, p)=\left\{T^{j}(s, p), j \in \mathbb{Z}\right\} \subset \mathcal{A} .
$$

A billiard has no fixed points. However, given $n \geq 2$, Birkhoff's Theorem states that $T$ has at least two different orbits of period $n$ which will be fixed points of $T^{n}$. The linearization of $T^{n}$ at any of these fixed points, say $(s, p)$, gives the linear area preserving map $D T_{(s, p)}^{n}$, which has a fixed point at the origin $(0,0)$. According to the eigenvalues of this linear map, the fixed point $(s, p)$ is classified as: hyperbolic if the eigenvalues of are $\mu$ and $\frac{1}{\mu}, \mu \in \mathbb{R}, \mu \neq \pm 1$, elliptic if the eigenvalues are $\mu=e^{i \gamma}$ and $\bar{\mu}, \mu^{2} \neq 1$ or parabolic if the eigenvalues are 1 or -1 .

In the hyperbolic case, the Hartman-Grobman Theorem (see, for instance, [8] or [13]) assures that, on a neighbourhood of the fixed point $(s, p)$, the dynamical behaviour of $T^{n}$ is the same as the dynamical behaviour of $D T_{(s, p)}^{n}$ on a neighbourhood of the origin. So, $(s, p)$ is an unstable fixed point of $T^{n}$ and $\left\{(s, p), T(s, p), \ldots, T^{n-1}(s, p)\right\}$ is an unstable periodic orbit of $T$. In this case, the instability of the equilibrium of the linear map $D T_{(s, p)}^{n}$ implies the local instability of the periodic orbit for the complete map $T$.

In the elliptic case, the linear map $D T_{(s, p)}^{n}$ is a rotation: the origin is surrounded by closed invariant circles and is a stable equilibrium. However, this beautiful behaviour may not be inherited by the map $T^{n}$, as it can be seen in the examples on the figure bellow. For both of them, the fixed point is linearly elliptic. On the left side, the non linear map exhibit invariant closed curves surrounding the fixed point, which is then stable. For the non linear map on the right, no invariant curves can be observed and the fixed point seems to be unstable. ${ }^{1}$

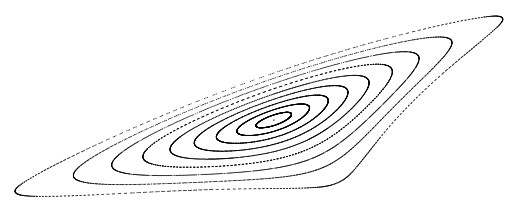

Moreover, it is not even clear if the pictures above, obtained by numerical simulations, correspond to the true behaviour of the maps. In fact, Dias Carneiro and ourselves [5] have proved that any $C^{1}$ strictly convex billiard map with

\footnotetext{
${ }^{1}$ Even more surprising is the example given by Anosov and Katok in [1] of an ergodic area-preserving map of the disc $|z|<1$, with an elliptic fixed point at $z=0$. The ergodicity implies that the fixed point is unstable. This example does not represent a billiard map and we don't know if there are any billiards with this property.
} 
an elliptic 2-periodic orbit can be approached by billiard maps with a 2-periodic orbit surrounded by closed invariant curves, ie, with a stable orbit. We guess this result can be extended to any period. Therefore, because of natural numerical round off errors, one can not be sure that the simulation corresponds to the actual billiard at and not to a very close one.

As a consequence, in the elliptic case, a more carefull approach is needed and higher order terms must be taken into account to assure the local stability of periodic orbits. A classical way to handle this problem is to use the Birkhoff Normal Form and Moser's Twist Theorem [14.

In what follows we explain how this can be performed and applied to the billiard map in the case of 2-periodic elliptic orbits. We have employed the software (BMaple to calculate the necessary data and all the worksheets are available at http://www.mat.ufmg.br/ syok/papers We then apply the results to two special classes of billiards.

Related works are Hayli, Dumont, Moulin-Ollagnier, Strelcyn [7] and Moeckel [1]. The first authors studied the stability of periodic orbits for a family of Robnik's billiards. The last author studied the generic behaviour of the first Birkhoff coefficient for one-parameter families of conservative maps.

\section{Non linear analysis and the local stability of elliptic orbits}

Let $T$ be an area preserving map with a $n$-periodic orbit $\left\{(0,0), T(0,0), \ldots, T^{n-1}(0,0)\right\}$. We will assume that the map is $C^{k}$ with $k \geq 4$. In the case of the billiard map, this is equivalent to assume that the curve $\alpha$ is at least $C^{5}$.

The map $T^{n}$ can then be expanded in Taylor Form up to order 3 in a neighbourhood of its fixed point $(0,0)$,

$$
T^{n}(s, p)=\left(a_{10} s+a_{01} p+a_{20} s^{2}+\ldots+a_{03} p^{3}, b_{10} s+b_{01} p+b_{20} s^{2}+\ldots+b_{03} p^{3}\right)+\mathcal{O}\left(|(s, p)|^{4}\right) .
$$

If the fixed point is elliptic, with eigenvalues $\mu=\cos \gamma+i \sin \gamma$ and $\bar{\mu}$, by means of a complex linear area preserving coordinate change which diagonalizes the linear part, the map $T^{n}$ can be written as

$$
z \mapsto \mu\left(z+c_{20} z^{2}+c_{11} z \bar{z}+c_{02} \bar{z}^{2}+c_{30} z^{3}+c_{21} z^{2} \bar{z}+c_{12} z \bar{z}^{2}+c_{03} \bar{z}^{3}\right)+\mathcal{O}\left(|z|^{4}\right) .
$$

If $\mu^{j} \neq 1, j=1,2,3$ or 4 we say that $\mu$ is non resonant and an analytic coordinate change brings the map into its convergent Birkhoff Normal Form

$$
z \mapsto \mathrm{e}^{i\left(\gamma+\tau_{1}|z|^{2}\right)} z+\mathcal{O}\left(|z|^{4}\right)=\mu z+i \mu \tau_{1} z|z|^{2}+\mathcal{O}\left(|z|^{4}\right) .
$$

The first Birkoff coefficient $\tau_{1}$ is given by

$$
\tau_{1}=\Im\left(c_{21}\right)+\frac{\sin \gamma}{\cos \gamma-1}\left(3\left|c_{20}\right|^{2}+\frac{2 \cos \gamma-1}{2 \cos \gamma+1}\left|c_{02}\right|^{2}\right)
$$

where $\Im\left(c_{21}\right)$ stands for the imaginary part of $c_{21}$.

The calculations leading to expression (4) are standard (17, 11]) and can also be easily performed using symbolic programming. ${ }^{2}$

By Moser's Twist Theorem if the first Birkhoff coefficient $\tau_{1}$ is not zero there are $T^{n}$-invariant curves surrounding the fixed point and therefore it is stable. We have that each point of the n-periodic orbit is contained in an open set, called an island, homeomorphic to a disk and invariant under $T^{n}$. Each island contains $T^{n}$-invariant curves surrounding the periodic point. So, the n-periodic orbit of $T$ is stable.

\footnotetext{
${ }^{2}$ http://www.mat.ufmg.br/ syok/papers/3NormalForm.
} 


\section{Elliptic 2-periodic orbits of convex billiards}

Any closed regular strictly convex $C^{2}$ curve $\alpha$ has at least two diameters, characterized by points with parallel tangents and equal normal lines (like the axis of an ellipse). The motion along each one of them corresponds to a 2-periodic trajectory for the billiard map associated to $\alpha$.

It is easy to prove that the longest of these diameters, if isolated, corresponds to a hyperbolic orbit (see, for instance [8] or 9]). The other(s) can be either hyperbolic, elliptic or parabolic. Let us suppose that one of them is elliptic and let $s=0$ and $s=s_{1}$ be the arclength parameters of the trajectory. As the motion occurs in the normal direction, the tangential component of the momentum $p$ is zero in both of the reflection points. Then $\left\{(0,0),\left(s_{1}, 0\right)\right\}$ is an elliptic 2-periodic orbit of the associated billiard map $T(s, p)=(S(s, p), P(s, p))$ and $(0,0)$ is an elliptic fixed point of $T^{2}$.

Let $R_{0}=R(0)$ and $R_{1}=R\left(s_{1}\right)$ be the radii of curvature of $\alpha$ at the $s=0$ and $s=s_{1}$ and $L=\left\|\alpha(0)-\alpha\left(s_{1}\right)\right\|$ be the length of the trajectory. Using equation (1), the linear map $D T_{(0,0)}^{2}=D T_{\left(s_{1}, 0\right)} D T_{(0,0)}$ is given by

$$
D T_{(0,0)}^{2}=\left(\begin{array}{cc}
\frac{\left(L-R_{1}\right)\left(L-R_{0}\right)}{R_{1} R_{0}}+\frac{L\left(L-R_{0}-R_{1}\right)}{R_{0} R_{1}} & -\frac{2 L\left(L-R_{1}\right)}{R_{1}} \\
-\frac{2\left(L-R_{0}\right)\left(L-R_{0}-R_{1}\right)}{R_{0}{ }^{2} R_{1}} & \frac{\left(L-R_{1}\right)\left(L-R_{0}\right)}{R_{1} R_{0}}+\frac{L\left(L-R_{0}-R_{1}\right)}{R_{0} R_{1}}
\end{array}\right)
$$

and its eigenvalues are

$$
2 \frac{\left(L-R_{1}\right)\left(L-R_{0}\right)}{R_{0} R_{1}}-1 \pm \frac{2 \sqrt{L\left(L-R_{0}-R_{1}\right)\left(L-R_{1}\right)\left(L-R_{0}\right)}}{R_{0} R_{1}}
$$

As the trajectory is elliptic the relations $L-R_{0}-R_{1}<0$ and $\left(L-R_{0}\right)\left(L-R_{1}\right)>0$ must be fullfilled. Assuming that $4\left(L-R_{0}\right)\left(L-R_{1}\right) \neq R_{0} R_{1}$ and $2\left(L-R_{0}\right)\left(L-R_{1}\right) \neq R_{0} R_{1}$ then $\mu^{j} \neq 1$ for $j=1,2,3,4$.

In the elliptic and non-resonant case, in order to investigate the stability of the fixed point, we can proceed and examine the first Birkhoff coefficient given by (4) . The complex coefficients $c_{21}, c_{20}$ and $c_{02}$ in the formula depend on the real coefficients $a_{i j}$ and $b_{i j}$ of the Taylor expansion of $T^{2}$ at the origin (2).

The linear coefficients $a_{i j}$ and $b_{i j}, i+j=1$, are obviously the entries of $D T_{(0,0)}^{2}$ and thus given by (5). Note that $a_{10}=b_{01}$. As $T$ is area preserving, $a_{10}^{2}-a_{01} b_{10}=1$ and, as $(0,0)$ is elliptic, $a_{01} b_{10}<0$.

These conditions were used to write down the coordinate change leading to (3) and we got ${ }^{3}$ :

$$
\begin{aligned}
& \Im\left(c_{21}\right)=\frac{a_{10}}{8}\left(-a_{21}+3 \frac{b_{10}}{a_{01}} a_{03}-3 \frac{a_{01}}{b_{10}} b_{30}+b_{12}\right)-\frac{b_{10}}{8}\left(a_{12}-3 \frac{a_{01}}{b_{10}} a_{30}-\frac{a_{01}}{b_{10}} b_{21}+3 b_{03}\right) \\
& \left|c_{20}\right|^{2}=\frac{1}{16} \sqrt{-\frac{a_{01}}{b_{10}}}\left(\frac{b_{10}}{a_{01}} a_{02}+a_{20}+b_{11}\right)^{2}+\frac{1}{16} \sqrt{-\frac{b_{10}}{a_{01}}}\left(\frac{a_{01}}{b_{10}} b_{20}+b_{02}+a_{11}\right)^{2} \\
& \left|c_{02}\right|^{2}=\frac{1}{16} \sqrt{-\frac{a_{01}}{b_{10}}}\left(\frac{b_{10}}{a_{01}} a_{02}+a_{20}-b_{11}\right)^{2}+\frac{1}{16} \sqrt{-\frac{b_{10}}{a_{01}}}\left(\frac{a_{01}}{b_{10}} b_{20}+b_{02}-a_{11}\right)^{2}
\end{aligned}
$$

which shows that $\tau_{1}$ is linear on the real coefficients of third order and quadratic on the second order ones.

In order to calculate explicitly the first Birkhoff coefficient, all is needed now are the second and third order coefficients of the Taylor expansion at $(0,0)$ of $T^{2}(s, p)=(S(S(s, p), P(s, p)), P(S(s, p), P(s, p)))$.

\footnotetext{
${ }^{3}$ http://www.mat.ufmg.br/ $\sim$ syok/papers/2Complex.
} 
A sequence of straightforward but long computations using the Chain Rule gives those Taylor coefficients. To illustrate it, let us give the expression of $a_{20}$

$$
\begin{aligned}
a_{20}= & \frac{\partial^{2}}{\partial s^{2}} S(S(s, p), P(s, p))(0,0) \\
= & \frac{\partial S}{\partial s}(0,0) \frac{\partial P}{\partial s}(0,0) \frac{\partial^{2} S}{\partial s \partial p}\left(s_{1}, 0\right)+\frac{1}{2} \frac{\partial S}{\partial s}\left(s_{1}, 0\right) \frac{\partial^{2} S}{\partial s^{2}}(0,0)+\frac{1}{2}\left[\frac{\partial P}{\partial s}(0,0)\right]^{2} \frac{\partial^{2} S}{\partial p^{2}}\left(s_{1}, 0\right)+ \\
& +\frac{1}{2} \frac{\partial S}{\partial p}\left(s_{1}, 0\right) \frac{\partial^{2} P}{\partial s^{2}}(0,0)+\frac{1}{2}\left[\frac{\partial S}{\partial s}(0,0)\right]^{2} \frac{\partial^{2} S}{\partial s^{2}}\left(s_{1}, 0\right)
\end{aligned}
$$

The first derivatives of the functions $S$ and $P$ are given by formulae (1)nd they depend on the function $l(s, p)$. So, to calculate he second and third derivatives of $S$ and $P$ it is necessary to evaluate the first and second derivatives of $l$. Let $l(s, S)=\|\alpha(S)-\alpha(s)\|$. Then $l(s, p)=l(s, S(s, p))$.

By differentiating

$$
l^{2}(s, S)=\langle\alpha(S)-\alpha(s), \alpha(S)-\alpha(s)\rangle
$$

we have

$$
l(s, S) \frac{\partial l}{\partial s}(s, S)=-\left\langle\alpha^{\prime}(s), \alpha(S)-\alpha(s)\right\rangle
$$

and so, as $\alpha^{\prime}$ is the unitary tangent vector,

$$
\frac{\partial l}{\partial s}(s, S)=-p
$$

Analogously

$$
\frac{\partial l}{\partial S}(s, S)=P
$$

These relations simply shows that $-l(s, S)$ is the generating function of the billiard map, as a Twist map.

Differentiating (7) with respect to $s$ and $S$ and using that $\eta=R \alpha^{\prime \prime}$ is the unitary normal vector gives

$$
\begin{gathered}
\frac{\partial^{2} l}{\partial s^{2}}(s, S)=\frac{1-p^{2}}{l(s, S)}-\frac{\sqrt{1-p^{2}}}{R(s)} \\
\frac{\partial^{2} l}{\partial s \partial S}(s, S)=\frac{\sqrt{\left(1-p^{2}\right)\left(1-P^{2}\right)}}{l(s, S)} .
\end{gathered}
$$

The same reasoning gives

$$
\frac{\partial^{2} l}{\partial S^{2}}(s, S)=\frac{1-P^{2}}{l(s, S)}-\frac{\sqrt{1-P^{2}}}{R(S)}
$$

The chain rule will give, now, the first and second order derivatives of $l(s, p)$. To evaluate them at $(0,0)$ and $\left(s_{1}, 0\right)$ it is usefull to remember that $\alpha^{\prime}\left(s_{1}\right)=-\alpha^{\prime}(0), \eta\left(s_{1}\right)=-\eta(0)$ and $\alpha\left(s_{1}\right)-\alpha(0)=L \eta(0)$.

Because of the recurrent structure of the formulae, the explicit calculus of the $a_{i j}$ and $b_{i j}$ is suitable to be implemented as a computer program ${ }^{4}$. The final expression of the Taylor expansion of $T^{2}$ is also given in the worksheet 1TaylorCoeffs.

The second order coefficients $a_{i j}, b_{i j}, i+j=2$ will have linear dependence on $\frac{d R}{d s}(0)=R_{0}^{\prime}$ and $\frac{d R}{d s}\left(s_{1}\right)=R_{1}^{\prime}$ while the third order coefficients $a_{i j}, b_{i j}, i+j=3$ will have linear dependence on $\frac{d^{2} R}{d s^{2}}(0)=R_{0}^{\prime \prime}$ and $\frac{d^{2} R}{d s^{2}}\left(s_{1}\right)=R_{1}^{\prime \prime}$ and quadratic on the first order derivatives. So the first Birkhoff coefficient $\tau_{1}$ will be quadratic on the first derivatives of $R$ and

\footnotetext{
${ }^{4}$ http://www.mat.ufmg.br/ syok/papers/0ThreeJet and 1TaylorCoeffs
} 
linear on the second ones. The final expression of $\tau_{1}$ is obtained after substitution of the $a_{i j}$ and $b_{i j}$ into (7) and then into (41) given

$$
\begin{aligned}
\tau_{1}= & -\frac{1}{8} \frac{R_{0}+R_{1}}{R_{0} R_{1}}-\frac{1}{8} \frac{L}{L-R_{0}-R_{1}}\left(\frac{L-R_{1}}{L-R_{0}} R_{0}^{\prime \prime}+\frac{L-R_{0}}{L-R_{1}} R_{1}^{\prime \prime}\right) \\
& -\frac{1}{8} \frac{L}{\left(L-R_{0}-R_{1}\right)^{2}}\left(2 \frac{L-R_{1}}{L-R_{0}}\left(R_{0}^{\prime}\right)^{2}+2 \frac{L-R_{0}}{L-R_{1}}\left(R_{1}^{\prime}\right)^{2}+3 R_{0}^{\prime} R_{1}^{\prime}\right) \\
& +\frac{1}{8} \frac{L R_{0} R_{1}}{\left(L-R_{0}-R_{1}\right)^{2}\left(4\left(L-R_{0}\right)\left(L-R_{1}\right)-R_{0} R_{1}\right)}\left(\frac{\left(L-R_{1}\right)^{2}}{L-R_{0}} \frac{\left(R_{0}^{\prime}\right)^{2}}{R_{0}}+\frac{\left(L-R_{0}\right)^{2}}{L-R_{1}} \frac{\left(R_{1}^{\prime}\right)^{2}}{R_{1}}-R_{0}^{\prime} R_{1}^{\prime}\right)
\end{aligned}
$$

The details leading to the formula are given in the worksheet 4 Tau.

\section{Billiards with islands}

As remarked in section 3 a billiard on a strictly convex $C^{2}$ curve always has 2-periodic orbits and the largest one, if isolated, is hyperbolic. Unfortunately, one cannot assure that at least one of the others is elliptic. In fact, there are many examples where all 2-periodic orbits are isolated and hyperbolic (see, for instance [5] or [9]).

On the other hand, ellipticity is an open property, in the sense that if a billiard associated to a $C^{2}$ strictly convex curve $\alpha$ has an elliptic 2-periodic orbit, then any strictly convex curve sufficiently $C^{2}$-close to $\alpha$ generates a billiard with an elliptic 2-periodic orbit [5]. So, a large class of strictly convex billiards has elliptic 2-periodic orbits. The question is: are they stable?

In what follows we present two classes of billiards (locally circular and symmetric) exhibiting stable 2-periodic orbits.

\subsection{Locally Circular Billiards}

Our first and simplest example is a 2-periodic orbit between two circles. More precisely, let $\alpha$ be a $C^{5}$ plane strictly convex closed curve parameterized by the arclength $s$, with the following properties:

- there are two points located by $s=0$ and $s=s_{1}$ such that $\alpha^{\prime}(0)=-\alpha^{\prime}\left(s_{1}\right)$ and $\alpha(0)-\alpha\left(s_{1}\right)=-L \vec{\eta}(0)$, where $\vec{\eta}(0)$ is the unitary normal vector at 0 .

- $\alpha$ is locally a circle, both near $s=0$ and $s=s_{1}$, with radii $R_{0}$ and $R_{1}$ respectively.

- $L, R_{0}$ and $R_{1}$ verify $L-R_{0}-R_{1}<0,\left(L-R_{0}\right)\left(L-R_{1}\right)>0$ and $4\left(L-R_{0}\right)\left(L-R_{1}\right) \neq R_{0} R_{1}, 2 R_{0} R_{1}$, which are open conditions on the $\left(L, R_{0}, R_{1}\right)$-space.

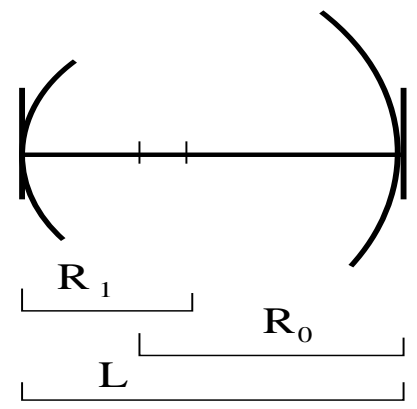


With these properties, $\left\{(0,0),\left(s_{1}, 0\right)\right\}$ is a non-resonant elliptic 2-periodic orbit for the billiard map $T$ associated to $\alpha$.

As $\alpha$ is locally circles, $T$ is locally analytic and the first Birkhoff coefficient of the elliptic orbit can be calculated. Moreover, $R^{\prime}$ and $R^{\prime \prime}$ vanish at $s=0$ and $s=s_{1}$. So,

$$
\tau_{1}=-\frac{1}{8}\left(\frac{1}{R_{0}}+\frac{1}{R_{1}}\right) \neq 0
$$

and this billiard has a stable 2-periodic orbit.

Although extremely simple, this example shows that exchanging the curve $\alpha$ by the osculating circles at the impact points gives information about ellipticity, but not about stability, since $\tau_{1}$ depends on the derivatives of the radius of curvature.

\subsection{Ovals with a special symmetry}

Let $R$ be a periodic $C^{4}$ function with Fourier expansion

$$
R(\varphi)=a_{0}+\sum_{n=1} a_{n} \cos 2 n \varphi
$$

with $a_{n}>0$ and $a_{0}>\sum_{n=1} a_{n}$, implying that $R(\varphi)>0, \forall \varphi$.

Let $\alpha$ be a curve, having $R$ as its radius of curvature, given by

$$
\alpha(\varphi)=(x(\varphi), y(\varphi))=\left(\int_{0}^{\varphi} R(\beta) \cos \beta d \beta, \int_{0}^{\varphi} R(\beta) \sin \beta d \beta\right) .
$$

It is a regular, closed and strictly convex $C^{5}$ curve.

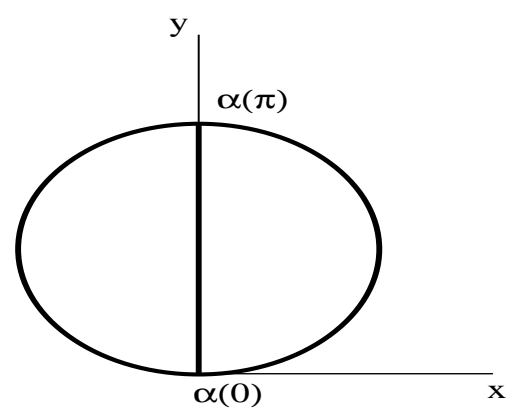

As $R$ is an even function, $x(-\varphi)=-x(\varphi), y(-\varphi)=y(\varphi)$ and $\overline{\alpha(0) \alpha(\pi)}$ is an axis of symmetry for $\alpha$. $\{(0,0),(\pi, 0)\}$ is a 2 -periodic orbit for the associated billiard map.

We have

$$
\begin{gathered}
L=\|\alpha(\pi)-\alpha(0)\|=y(\pi)=2 a_{0}-\sum_{n=1} \frac{2 a_{n}}{(2 n+1)(2 n-1)} \\
R(0)=R(\pi)=R_{0}=a_{0}+\sum_{n=1} a_{n}
\end{gathered}
$$


and then

$$
\begin{aligned}
L-R(0)-R(\pi)=L-2 R_{0} & =-2 \sum_{n=1} a_{n}\left(\frac{1}{(2 n+1)(2 n-1)}+1\right)<0 \\
L-R(0)=L-R(\pi)=L-R_{0} & =a_{0}-\sum_{n=1} a_{n}\left(\frac{2}{(2 n+1)(2 n-1)}+1\right)
\end{aligned}
$$

If

$$
(2+k) a_{0}-\sum_{n=1} a_{n}\left[\frac{4}{(2 n+1)(2 n-1)}+(2-k)\right] \neq 0, \quad k=0, \pm 1, \pm \sqrt{2}
$$

which are open conditions, then $\{(0,0),(\pi, 0)\}$ is elliptic and non-resonant.

Let $s=s(\varphi)$ be the arclength parameter for $\alpha$. Choosing $s(0)=0$ and $s(\pi)=s_{1}$ we have

$$
\begin{gathered}
\left.\frac{d R}{d s}\right|_{s=0}=\left.\frac{1}{R_{0}} \frac{d R}{d \varphi}\right|_{\varphi=0}=0 \quad,\left.\quad \frac{d R}{d s}\right|_{s=s_{1}}=\left.\frac{1}{R_{0}} \frac{d R}{d \varphi}\right|_{\varphi=\pi}=0 \\
\left.\frac{d^{2} R}{d s^{2}}\right|_{s=0}=\left.\frac{1}{R_{0}^{2}} \frac{d^{2} R}{d \varphi^{2}}\right|_{\varphi=0}=-4 \sum_{n=1} n^{2} a_{n}<0 \quad,\left.\quad \frac{d^{2} R}{d s^{2}}\left(s_{1}\right)\right|_{s=s_{1}}=\left.\frac{1}{R_{0}^{2}} \frac{d^{2} R}{d \varphi^{2}}\right|_{\varphi=\pi}=-4 \sum_{n=1} n^{2} a_{n}<0
\end{gathered}
$$

and the first Birkhoff coefficient is

$$
\tau_{1}=-\frac{1}{4 R_{0}}\left(1+\frac{L}{R_{0}\left(L-2 R_{0}\right)} \frac{d^{2} R}{d \varphi^{2}}(0)\right)<0
$$

So the 2-periodic orbit is stable.

In particular, this class of curves include those studied numerically by Berry in 2 and defined by $R(\varphi)=1+\epsilon \cos 2 \varphi$ with $0<\epsilon<1$. If $\epsilon \neq \frac{3}{5}, \frac{3}{13}$ or $\frac{3}{41}(13-8 \sqrt{2})$, the conditions for non-resonant ellipticity are fullfilled and the 2-periodic orbit is stable. It is claimed in [2] that when $\epsilon=\frac{3}{5}$ (meaning parabolicity of the 2-periodic orbit) there is neutral stability. There is no specific observations for the other two values of $\epsilon$.

It would be interesting to investigate the behaviour of this and other examples at resonances.

Acknowledgments The authors would like to thank M. J. Dias Carneiro for many enlightening discussions. This work is supported by CNPq and FAPEMIG brazilian agencies.

\section{References}

[1] D. V. Anosov, A. B. Katok: New examples in smooth ergodic theory, Ergodic Diffeomorphisms, Trudy Mosk. Math. Obs. 23, 3-36 (1970), engl.transl. in Trans. Mosc. Math. Soc., AMS 23, 1-35 (1972).

[2] M. V. Berry: Regularity and chaos in classical mechanics, illustrated by three deformations of a circular "billiard". European J. Phys. 2/2, 91-102 (1981)

[3] G. D. Birkhoff: Dynamical Systems, AMS Coll.Pub., 1966 (Original ed. 1927)

[4] N. Chernov, R. Markarian: Introduction to the Ergodic Theory of Chaotic Billiards, Pub.Mat. IMPA-RJ, 2003.

[5] M. J. Dias Carneiro, S. Oliffson Kamphorst, S. Pinto de Carvalho: Elliptic Islands in Strictly Convex Billiards, Erg. Th. Dyn. Sys. 23/3, 799-812 (2003). 
[6] B. Hasselblat, A. Katok: A First Course in Dynamics, Cambridge Univ.Press, 2002.

[7] A. Hayli, T. Dumont, J. Moulin-Ollagnier, J.-M. Strelcyn: Quelques résultats nouveaux sur les billards de Robnik, J. Phys. A 20/11, 3237-3249 (1987).

[8] A. Katok, B. Hasselblat: Introduction to the Modern Theory of Dynamical Systems, Cambridge Univ.Press, 1995.

[9] V. V. Kozlov: Two-link billiard trajectories: extremal properties and stability,(Russian) Prikl. Mat. Mekh. 64/6, 942-946 (2000). ; translation in J. Appl. Math. Mech. 64/6 903-907 (2001)

[10] V.V. Kozlov, D.V. Treshchëv: Billiards: A Genetic Introduction to the Dynamics of Systems with Impacts, Transl. Math. Monog., AMS, 1991.

[11] R. Moeckel: Generic bifurcations of the twist coefficient, Erg. Th. Dyn. Syst. 10, 185-195 (1990)

[12] J. Moser: Stable and random motions in dynamical systems, PUP, 1973.

[13] J. Palis Jr., W. de Melo: Introdução aos sistemas dinâmicos, Proj. Euclides, IMPA-RJ, 1978.

[14] C. L. Siegel, J. K. Moser: Lectures on Celestial Mechanics, Springer-Verlag, 1971.

[15] J-M. Strelcyn: Plane Billiards as Smooth Dynamical Systems with Singularities. In: Katok, Strelcyn, in coll. with Ledrappier, Przytycki, LNM 1222, Springer-Verlag, 1986

[16] S. Tabachnikov: Billiards, Panoramas et Synthèses, SMF, 1995. 